\title{
A complexidade da tarefa afeta negativamente 0 equilíbrio e a mobilidade de idosos saudáveis
}

\author{
Negative effect of task complexity on the balance and mobility of healthy older adults
}

Gabriella Simões Scarmagnan' (DD Sarah Cristina Mugica de $\mathrm{Mell}_{0}{ }^{2}$ (D) Tayla Borges Lino ${ }^{3}$ ID Fabio Augusto Barbieri ${ }^{4}$ (D) Gustavo Christofoletti ${ }^{1,2,3}$ (D)

\section{Resumo}

Objetivo: Analisar o impacto da complexidade da tarefa sobre mobilidade e equilíbrio de idosos saudáveis. Métodos: Noventa idosos foram incluídos neste estudo. Os participantes foram submetidos a tarefas que simularam problemas comuns ao envelhecimento, como baixa acuidade visual, alterações na base de apoio e dificuldades em realizar atividades simultâneas. Para avaliação da mobilidade utilizou-se o teste Timed Get Up and Go associado a atividades de dupla-tarefa cognitiva e motora. Para o equilíbrio utilizou-se uma plataforma de força avaliada em diferentes bases de apoio e informações visuais. Testes cognitivos foram aplicados com objetivo de caracterizar a amostra e analisar a associação entre as variáveis motoras e cognitivas. Na análise estatística utilizou-se o teste de Friedman para verificar o efeito da complexidade da tarefa nos idosos e o coeficiente de correlação de Spearman para verificar a associação entre as variáveis motoras e cognitivas. O nível de significância foi estipulado em 5\%. Resultados: A complexidade da atividade interferiu na mobilidade dos participantes, exigindo um maior número de passos e tempo para realizar a tarefa $(p=0,001)$. De maneira semelhante, uma base de apoio diminuída e informações visuais restritas causaram uma maior insegurança nos participantes frente às reações de equilíbrio $(p=0,001)$. Testes de correlação apontaram associações significativas entre funções executivas e tarefas motoras complexas $(\not<0,05)$.

\footnotetext{
Universidade Federal de Mato Grosso do Sul (UFMS), Faculdade de Medicina, Programa de Pós-Graduação em Saúde e Desenvolvimento da Região Centro-Oeste. Campo Grande, MS, Brasil.

2 Universidade Federal de Mato Grosso do Sul (UFMS), Instituto de Saúde, curso de Fisioterapia. Campo Grande, MS, Brasil.

3 Universidade Federal de Mato Grosso do Sul (UFMS), Instituto de Saúde, Programa de pós-graduação em Ciências do Movimento. Campo Grande, MS, Brasil.

4 Universidade Estadual Paulista (UNESP), Departamento de Educação Física, Programa de pós-graduação em Ciências da Motricidade - Interunidades. Bauru, SP, Brasil.
}

Financiamento da pesquisa: Coordenação de Aperfeiçoamento de Pessoal de Nível Superior (CAPES - código 001) e Pró-reitoria de Pesquisa e Pós-graduação da Fundação Universidade Federal de Mato Grosso do Sul (PROPP/UFMS)

Os autores declaram não haver conflito na concepção deste trabalho.

Palavras-chave: Saúde do Idoso. Equilíbrio Postural. Limitação da Mobilidade. Acidentes por Quedas. Cognição. 
Conclusão: Idosos saudáveis apresentam instabilidade motora quando submetidos a tarefas complexas, potencializando as alterações provenientes do envelhecimento. A associação entre as variáveis cognitivas e motoras sugere a necessidade de assistência multiprofissional para preparar os idosos no enfrentamento de desafios diários.

\section{Abstract}

Objective: to analyze the impact of task complexity on the mobility and balance of healthy older adults. Methods: ninety older adults were enrolled in the study. The participants performed tasks that simulated problems common to aging, such as low visual acuity, changes in the base of support and difficulties in carrying out activities simultaneously. Mobility was evaluated with the Timed Get Up and Go test during dual cognitive and motor tasks. Balance was assessed using a force plate with different bases of support and visual information. Cognitive tests were applied to characterize the sample and to analyze the association between the motor and cognitive variables. For statistical analysis, the Friedman test was used to verify the impact of task complexity on the older adults and the Spearman correlation coefficient was used to verify the association between the motor and cognitive variables. Significance was set at $5 \%$. Results: task complexity impacted the mobility of participants, with a greater number of steps and time required to complete the test $(p=0.001)$. Similarly, small bases of support and restricted visual information resulted in greater insecurity among the participants in terms of balance reactions $(p=0.001)$. Correlation tests identified significant associations between executive functions and complex motor tasks $(p<0.05)$. Conclusion: healthy older adults exhibited motor instability when performing complex tasks, potentiating aging-related changes. The association between the cognitive and motor variables suggests the need of multiprofessional care to prepare older adults for their daily challenges.
Keywords: Health of the Elderly. Postural Balance. Mobility Limitation. Accidental Falls. Cognition.

\section{INTRODUÇÃO}

O processo de envelhecimento é associado a uma série de transformações no organismo que nem sempre possibilitam ao indivíduo a independência necessária para realizar as suas atividades rotineiras ${ }^{1}$. Diante disso, a pessoa idosa percebe que não apresenta a mesma destreza e habilidade motora que antigamente e o processamento cognitivo não acompanha a demanda crescente de informação e decisão que a sociedade atual está sujeita ${ }^{2}$. Assim, o idoso se encontra em situações conflituosas e, sem perceber, muitas vezes se submete a condições que causam riscos à sua saúde 3 .

Nesse cenário, diversas pesquisas vêm sendo realizadas com o objetivo de analisar o impacto do envelhecimento sobre a saúde das pessoas. Problemas de fragilidades, equilíbrio, coordenação motora e força muscular têm sido já bem relatados na literatura ${ }^{4-6}$. A influência da cognição nas tarefas diárias tem sido investigada nos últimos anos mas uma parte significativa dos estudos concentra suas análises em idosos com demência - situação onde o declínio cognitivo é proeminente e patológico ${ }^{7,8}$.

A complexidade da tarefa é um aspecto importante a ser analisado na rotina dos idosos. Idosos saudáveis são submetidos a demandas cognitivas durante atividades diárias e que causam a divisão do foco da atenção ${ }^{9}$. O aparato cognitivo precisa estar preservado nos idosos porque situações complexas exigem atenção, concentração e processamento cognitivo para a sua execução. Esses aspectos tendem a sofrer influência do envelhecimento e afetar a saúde dos idosos ${ }^{10}$.

O entendimento das alterações que ocorrem no organismo é importante para analisar o impacto do envelhecimento sobre a rotina dos idosos, prevenindo complicações e riscos diversos. Neste estudo, idosos saudáveis foram submetidos a tarefas complexas que potencializaram alterações comuns ao envelhecimento, como baixa acuidade visual, alterações na base de apoio e dificuldades em realizar tarefas simultâneas. Com essa premissa, pretendeu-se 
avaliar o impacto que a complexidade da tarefa exerce sobre o aparato motor (mobilidade e equilíbrio) de idosos saudáveis.

A hipótese dos pesquisadores foi a de que a realização de atividades complexas que potencializam alterações comuns ao envelhecimento iria afetar a mobilidade e o equilíbrio dos idosos, gerando riscos à sua saúde. Tais informações poderão ser úteis para profissionais da saúde voltados à área da geriatria e gerontologia, uma vez que promove novas discussões sobre a relação do envelhecimento com atividades complexas diárias dos idosos.

\section{MÉTODOS}

Este estudo refere-se a uma pesquisa de delineamento transversal com dados quantitativos. A seleção dos participantes ocorreu de forma probabilística estratificada, tendo a idade e o sexo como critérios de seleção. Os participantes foram recrutados no município de Campo Grande/MS, e todos os sujeitos forneceram consentimento por escrito antes das avaliações. A pesquisa foi realizada em concordância com a Declaração de Helsinki e seu protocolo foi aprovado pelo Comitê de Ética institucional (protocolo número 2.305.644; CAAE: 73163817.2.0000.0021).

Os critérios de inclusão envolveram participantes de ambos os sexos, com idade igual ou superior a 60 anos, sem histórico de doenças neurológicas ou psiquiátricas, e com escores cognitivos no MiniExame do Estado Mental (MEEM) ${ }^{11}$ superiores à nota de corte estipulado por Brucki et al. ${ }^{12}$. Parâmetros normais no MEEM para a população brasileira são: mínimo de 20 pontos para pessoas iletradas; mínimo de 25 pontos para pessoas com 1 a 4 anos de escolaridade; mínimo de 26,5 pontos para pessoas com 5 a 8 anos de escolaridade; mínimo de 28 pontos para pessoas com 9 a 11 anos de escolaridade; e mínimo de 29 pontos para pessoas com escolaridade superior a 11 anos.

Os critérios de exclusão envolveram casos de vertigem, participantes que faziam uso de órteses ou próteses em membros inferiores, usuários de cadeira de rodas, pacientes acamados e aqueles que não conseguiram permanecer 60 segundos em posição ortostática. Além disso, nenhum participante poderia ter sido hospitalizado ou se submetido a cirurgia nos últimos 6 meses. Idosos residentes em instituições de longa permanência também foram excluídos da pesquisa.

O tamanho da amostra foi calculado assumindo um poder estatístico de $80 \%$, com um erro do tipo I de $5 \%$ e um tamanho de efeito de $0,27^{13}$. A análise final demonstrou a necessidade de um mínimo de 83 participantes. Nesta pesquisa, 110 idosos foram originalmente recrutados. Desses, 9 pessoas não foram incluídas por não desejarem participar do estudo, 4 foram excluídos por possuírem próteses em membros inferiores e 7 por apresentarem escores cognitivos abaixo da nota de corte estipulada por Brucki et al. ${ }^{12}$. Assim, 90 idosos compuseram a amostra final da pesquisa - correspondendo a $8,4 \%$ acima do tamanho amostral mínimo necessário.

Todos os procedimentos metodológicos estão descritos em concordância com a iniciativa $\mathrm{STROBE}^{14}$. Os participantes foram submetidos a avaliação no Laboratório de Biomecânica do Instituto de Saúde da Universidade Federal de Mato Grosso do Sul, que envolveu no primeiro momento uma anamnese com perguntas sobre aspectos gerais como idade, escolaridade, estado civil, ocupação profissional, índice de massa corpórea e práticas de atividade física. No segundo momento os pesquisadores utilizaram-se de testes específicos para avaliação da cognição, da mobilidade, do risco de quedas e do equilíbrio dos participantes. Todos os testes foram aplicados aleatoriamente de acordo com a distribuição do quadrado latino ${ }^{15}$.

As funções cognitivas foram analisadas por meio do MEEM $^{11,12}$ e da Bateria de Avaliação Frontal $(\mathrm{BAF})^{16}$. O MEEM foi utilizado para avaliar a cognição geral dos participantes. Esse instrumento é composto por sete categorias específicas: orientação temporal, orientação espacial, registro de três palavras, atenção e cálculo, recordação imediata e tardia das três palavras, linguagem e prática visuoconstrutiva. O escore do MEEM vai de 0 a 30 pontos e as notas de corte adotadas neste estudo foram as delimitadas por Brucki et al. ${ }^{12}$ (especificações já detalhadas anteriormente). 
A BAF foi incluída para avaliar as funções executivas dos participantes. Esse instrumento aborda as seguintes habilidades cognitivas: reconhecimento de conceitos, flexibilidade léxica, programação motora, instruções conflitantes, controle inibitório e autonomia ambiental. $\mathrm{O}$ escore da BAF vai de 0 a 18 pontos e as notas de corte adotadas neste estudo foram as detalhadas por Beato et al..$^{17}$ : mínimo de 10 pontos para pessoas com 1 a 3 anos de escolaridade, mínimo de 12 pontos para pessoas com 4 a 7 anos de escolaridade, mínimo de 13 pontos para pessoas com 8 a 11 anos de escolaridade, e mínimo de 15 pontos para pessoas com escolaridade superior a 11 anos.

A avaliação da mobilidade foi realizada por meio do teste Timed Up and Go (TUG) ${ }^{18}$. O teste consiste na capacidade da pessoa de se levantar de uma cadeira, andar três metros, dar a volta e se sentar na cadeira. Um número maior de passos e tempo para concluir a tarefa indica um risco aumentado de quedas ${ }^{19}$. Neste estudo, o TUG foi aplicado de três maneiras distintas: $1^{\circ}$ ) teste normal, conforme desenvolvido Podsiadlo \& Richardson ${ }^{18} ; 2^{\circ}$ ) teste com dupla-tarefa motora, onde o participante realizou o teste segurando na mão dominante um copo com $100 \mathrm{ml}$ de água; e $3^{\circ}$ ) teste com dupla-tarefa cognitiva, onde o participante realizou o teste concomitante à nomeação aleatória de animais. Essa diferenciação foi realizada com o objetivo de analisar a mobilidade dos idosos em situações de tarefas simples e simultâneas. A ordem de aplicação dos testes foi aleatória para minimizar o efeito de aprendizado sobre os resultados.

Em adição à análise da mobilidade, os pesquisadores incluíram avaliação do risco de quedas dos participantes. Nesse estudo o risco de quedas foi mensurado pela Escala Internacional de Eficácia de Quedas (FES-I) ${ }^{20}$ e de acordo com o número de quedas ocorridas nos últimos doze meses. Queda foi explicada ao participante como qualquer desequilíbrio corporal acentuado que culminou no contato nãointencional do corpo com o solo. A FES-I é uma ferramenta de fácil e rápida aplicação que mede o nível de preocupação da pessoa com a queda durante atividades sociais e físicas dentro e fora de casa. Nesse instrumento, pontuações mais altas indicam maior preocupação dos entrevistados sobre quedas.
O equilíbrio postural foi avaliado por meio de uma plataforma de força (BIOMEC 400_V4, EMG System ${ }^{\circledR}$ ), composta por uma placa de 500 $\mathrm{mm}^{2}, 4$ células de carga e um sistema de calibração de $100 \mathrm{~Hz}$. A referida plataforma foi utilizada por sua habilidade de analisar o centro de gravidade dos idosos. Os participantes realizaram todos os testes com os pés descalços e foram instruídos a permanecer na plataforma por 60 segundos. $O$ tempo de 60 segundos foi escolhido por ser esse o período padronizado em testes clássicos que avaliam equilíbrio corporal de idosos ${ }^{21}$.

A avaliação do equilíbrio ocorreu nas variáveis deslocamento corporal $(\mathrm{cm})$, área $\left(\mathrm{cm}^{2}\right)$ e velocidade do deslocamento postural $(\mathrm{cm} / \mathrm{s})$. Os dados da plataforma de força foram processados no programa MATLAB $^{\circledR}$ (The Mathworks, Natick, MA). A rotina de dados foi delimitada para uma amostragem de 100 quadros por segundo, com filtro digital do tipo passa-baixa de Butterworth de $2^{a}$ ordem em $35 \mathrm{~Hz}$. Na plataforma de força, valores negativos nos planos anteroposterior e médio-lateral representam deslocamento do corpo para trás e para a esquerda, respectivamente.

As avaliações na plataforma de força envolveram quatro tarefas, diferindo em relação à informação visual (olhos abertos e olhos fechados) e base de apoio (bases de apoio de 30 e $10 \mathrm{~cm}$ ). O uso dessas condições teve como objetivo simular alterações comuns à idade, como baixa acuidade visual e base de apoio instável. Assim como o TUG, a ordem de aplicação das condições na plataforma de força foi aleatória, com o objetivo de minimizar o efeito de aprendizado nos resultados. Por motivos de segurança, dois pesquisadores permaneceram em cada lado dos participantes durante as avaliações, com o objetivo de evitar quedas.

Os testes de Shapiro-Wilk e de Levenne foram aplicados em todos os dados para analisar padrões de normalidade e homogeneidade de variâncias. Aqueles dados que contemplaram os parâmetros de normalidade e homogeneidade de variâncias foram analisados pela estatística paramétrica. Aqueles dados que não cumpriram os parâmetros de normalidade e 
homogeneidade de variâncias foram analisados pela estatística não-paramétrica.

Assim, os testes t de Student independente, $\mathrm{U}$ de Mann-Whitney e Fisher foram utilizados quando se desejou comparar as variáveis deste estudo (paramétricas e não-paramétricas) em relação aos homens e mulheres. O teste de Friedman foi aplicado para verificar o efeito da complexidade da tarefa nas variáveis mobilidade e equilíbrio - tendo o pós-teste de Wilcoxon sido utilizado para realizar comparações pareadas. O coeficiente de correlação de Spearman (rho) foi aplicado tendo a variável sexo como fator covariante. O objetivo dessa análise foi investigar a associação entre variáveis cognitivas e motoras, tendo a diferença entre sexo controlada na análise inferencial.
Para melhor visualização dos achados, a descrição das variáveis desse estudo está em número de eventos, porcentagem e média \pm desvio-padrão. Para todas as análises o nível de significância foi estabelecido em 5\%. Outliers foram identificados a partir de valores superiores a 3 intervalos interquartis, e foram excluídos das análises descritivas e inferenciais ${ }^{22}$

\section{RESULTADOS}

A tabela 1 demonstra informações dos participantes em relação a sexo, idade, escolaridade, estado civil, ocupação profissional, índice de massa corpórea, práticas de atividade física, cognição e risco de quedas.

Tabela 1. Características gerais dos participantes ( $\mathrm{n}=90)$. Campo Grande/MS, 2019.

\begin{tabular}{|c|c|c|c|}
\hline Variáveis & Homens & Mulheres & $p$ \\
\hline Tamanho amostral, $\%$ & 27,8 & 72,2 & 0,001 \\
\hline Idade, anos & $68,1 \pm 7,0$ & $68,6 \pm 7,3$ & 0,797 \\
\hline Escolaridade, $\%$ & & & 0,297 \\
\hline Ensino superior completo & 28,0 & 6,2 & \\
\hline Ensino superior incompleto & 8,0 & 7,7 & \\
\hline Ensino médio completo & 36,0 & 23,1 & \\
\hline Ensino médio incompleto & 0,0 & 0,0 & \\
\hline Ensino básico completo & 16,0 & 36,8 & \\
\hline Ensino básico incompleto & 12,0 & 6,2 & \\
\hline Estado civil, \% & & & 0,001 \\
\hline Solteiro & 4,0 & 15,4 & \\
\hline Casado & 72,0 & 41,5 & \\
\hline Divorciado & 4,0 & 13,8 & \\
\hline Viúvo & 12,0 & 27,7 & \\
\hline União estável & 8,0 & 1,6 & \\
\hline Índice de massa corpórea, $\mathrm{Kg} / \mathrm{m}^{2}$ & $27,1 \pm 4,2$ & $27,1 \pm 4,3$ & 0,981 \\
\hline Atividade física, $\%$ & & & 0,465 \\
\hline $\operatorname{Sim}$ & 56,0 & 66,2 & \\
\hline Não & 44,0 & 33,8 & \\
\hline MEEM, pts & $27,5 \pm 2,1$ & $26,7 \pm 2,3$ & 0,134 \\
\hline BAF, pts & $15,3 \pm 2,1$ & $14,2 \pm 2,4$ & 0,030 \\
\hline FES-I, pts & $25,0 \pm 5,3$ & $25,8 \pm 7,8$ & 0,993 \\
\hline Quedas & & & 0,064 \\
\hline Sim, $\%$ & 12,0 & 32,3 & \\
\hline Não, \% & 88,0 & 67,3 & \\
\hline
\end{tabular}

Os dados estão expressos em número de eventos (\%) e média \pm desvio padrão. Valores de $p$ do teste exato de Fisher para tamanho amostral, escolaridade, estado civil, ocupação profissional, atividade física e quedas nos últimos doze meses. Valores de $p$ do teste t de Student independente para idade e índice e massa corpórea. Valores de $p$ do teste U de Mann Whitney para MEEM, BAF e FES-I. 
A tabela 2 detalha a mobilidade dos participantes durante atividades simples e atividades simultâneas. Os resultados mostram as mulheres realizaram a atividade no mesmo tempo que os homens, mas com um maior número de passos. A análise do impacto da complexidade da tarefa sobre a mobilidade apontou interferência da dupla-tarefa cognitiva e motora nos participantes, com maior tempo e número de passos para realizar a atividade com dupla-tarefa cognitiva, seguida da atividade com dupla-tarefa motora.

A tabela 3 detalha o equilíbrio estático dos participantes durante as atividades que simulam baixa acuidade visual e bases de apoio restritas. Os resultados indicaram respostas semelhantes entre homens e mulheres para as diversas atividades realizadas, à exceção da área da base de apoio que foi maior nos homens do que nas mulheres. Análises complementares confirmam o impacto da acuidade visual e de base de apoio sobre o equilíbrio dos idosos.

A tabela 4 demonstra o coeficiente de correlação de Spearman entre as variáveis cognitivas e motoras. Os resultados apontaram associação significativa das funções executivas com os testes de mobilidade, mas frágeis associações com testes de equilíbrio.

Tabela 2. Impacto da complexidade da tarefa sobre a mobilidade dos participantes. $(\mathrm{n}=90)$. Campo Grande/ MS, 2019.

\begin{tabular}{lllll}
\hline Variáveis & Tarefa simples & Dupla-tarefa motora & Dupla-tarefa cognitiva & $p_{(\text {tarefa) }}$ \\
\hline Tempo & & & & \\
Homens & $10,2 \pm 2,2$ & $10,8 \pm 2,5$ & $13,8 \pm 5,4^{\mathrm{a}, \mathrm{b}}$ & 0,001 \\
Mulheres & $10,8 \pm 2,2$ & $11,1 \pm 2,7$ & $13,5 \pm 4,3^{\mathrm{a}, \mathrm{b}}$ & 0,001 \\
$p_{\text {(sexo) }}$ & 0,248 & 0,623 & 0,810 & --- \\
Número de passos & & & 0,011 \\
Homens & $13,4 \pm 2,6$ & $13,9 \pm 2,3$ & $14,0 \pm 3,3^{\mathrm{a}}$ & 0,001 \\
Mulheres & $15,2 \pm 2,2$ & $15,7 \pm 2,5^{\mathrm{a}}$ & $15,7 \pm 3,2$ & --- \\
\hline$p_{(\text {sexo) }}$ & 0,004 & 0,007 & 0,016 & \\
\hline Os
\end{tabular}

Os dados estão expressos em média \pm desvio padrão. Valores de $p$ do teste U-Mann Whitney na comparação entre sexo. Valores de $p$ do teste de Friedman na análise do impacto da complexidade de tarefa. Análises complementares foram realizadas pelo pós-teste de Wilcoxon. ${ }^{a}=$ significa diferença no mesmo grupo comparado com a tarefa simples. ${ }^{b}=$ significa diferença no mesmo grupo comparado com a dupla-tarefa motora.

Tabela 3. Impacto da complexidade da tarefa sobre o equilíbrio dos participantes. (n=90). Campo Grande/MS, 2019.

\begin{tabular}{|c|c|c|c|c|c|}
\hline Variáveis & BA30-OA & BA30-OF & BA10-OA & BA10-OF & $p_{(\text {tarefa) }}$ \\
\hline \multicolumn{6}{|c|}{ Posição AP (cm) } \\
\hline Homens & $-1,0 \pm 3,0$ & $-1,1 \pm 2,4$ & $-2,9 \pm 2,8^{a}$ & $-2,0 \pm 2,8^{b}$ & 0,001 \\
\hline Mulheres & $-1,4 \pm 2,5$ & $-2,2 \pm 2,9^{a}$ & $-2,4 \pm 2,5^{a}$ & $-2,3 \pm 3,2$ & 0,001 \\
\hline$p_{(\text {sexo })}$ & 0,311 & 0,060 & 0,405 & 0,452 & --- \\
\hline \multicolumn{6}{|c|}{ Posição ML (cm) } \\
\hline Homens & $-0,8 \pm 1,5$ & $-0,8 \pm 1,6$ & $-0,6 \pm 1,0$ & $-0,4 \pm 1,0$ & 0,299 \\
\hline Mulheres & $-1,2 \pm 1,3$ & $-1,3 \pm 1,4$ & $-0,9 \pm 0,9^{\mathrm{a}, \mathrm{b}}$ & $-0,9 \pm 1,1^{\mathrm{b}}$ & 0,001 \\
\hline$p_{(\text {(sexo) }}$ & 0,153 & 0,152 & 0,193 & 0,077 & --- \\
\hline \multicolumn{6}{|l|}{ Área $\left(\mathrm{cm}^{2}\right)$} \\
\hline Homens & $2,9 \pm 1,4$ & $3,7 \pm 2,1$ & $5,4 \pm 2,8^{\mathrm{a}, \mathrm{b}}$ & $9,8 \pm 6,9^{\mathrm{a}, \mathrm{b}, \mathrm{c}}$ & 0,001 \\
\hline Mulheres & $2,2 \pm 1,8$ & $2,3 \pm 1,4 f$ & $3,7 \pm 1,5^{\mathrm{a}, \mathrm{b}}$ & $5,6 \pm 3,3^{a, b, c}$ & 0,001 \\
\hline$p_{(\mathrm{sexo})}$ & 0,013 & 0,006 & 0,006 & 0,003 & --- \\
\hline
\end{tabular}


Continuação da Tabela 3

\begin{tabular}{|c|c|c|c|c|c|}
\hline Variáveis & BA30-OA & BA30-OF & BA10-OA & BA10-OF & $p_{\text {(tarefa) }}$ \\
\hline \multicolumn{6}{|c|}{ Velocidade AP $(\mathrm{cm} / \mathrm{s})$} \\
\hline Homens & $1,5 \pm 0,6$ & $1,7 \pm 0,6^{\mathrm{a}}$ & $1,4 \pm 0,4^{\mathrm{b}}$ & $2,0 \pm 0,8^{a, c}$ & 0,001 \\
\hline Mulheres & $1,3 \pm 0,3$ & $1,5 \pm 0,3$ & $1,4 \pm 0,3$ & $1,7 \pm 0,4^{\mathrm{a}}$ & 0,001 \\
\hline$p_{(\text {sexo) }}$ & 0,431 & 0,364 & 0,777 & 0,051 & --- \\
\hline \multicolumn{6}{|c|}{ Velocidade ML (cm/s) } \\
\hline Homens & $1,0 \pm 0,2$ & $1,0 \pm 0,3$ & $1,4 \pm 0,4^{\mathrm{a}, \mathrm{b}}$ & $2,0 \pm 0,7^{\mathrm{a}, \mathrm{b}, \mathrm{c}}$ & 0,001 \\
\hline Mulheres & $1,0 \pm 0,3$ & $1,0 \pm 0,2$ & $1,3 \pm 0,3^{\mathrm{a}, \mathrm{b}}$ & $1,6 \pm 0,4^{\mathrm{a}, \mathrm{b}}$ & 0,001 \\
\hline$p_{(\mathrm{sexo})}$ & 0,788 & 0,555 & 0,651 & 0,035 & --- \\
\hline
\end{tabular}

BA30-OA: Base de apoio de $30 \mathrm{~cm}$, olhos abertos. BA30-OF: Base de apoio de $30 \mathrm{~cm}$, olhos fechados. BA10-OA: Base de apoio de $10 \mathrm{~cm}$, olhos abertos. BA10-OF: Base de apoio de $10 \mathrm{~cm}$, olhos fechados. AP: anteroposterior. ML: médio-lateral. Os dados estão expressos em média \pm desvio padrão. Valores de $p$ do teste U-Mann Whitney na comparação entre sexo. Valores de $p$ do teste de Friedman na análise do impacto da complexidade de tarefa. Análises complementares foram realizadas pelo pós-teste de Wilcoxon. ${ }^{a}=$ diferença no mesmo grupo comparado com BA30-OA; ${ }^{\mathrm{b}}=$ diferença no mesmo grupo comparado com BA30-OF; ${ }^{\mathrm{c}}=$ diferença no mesmo grupo comparado com BA10-OA.

Tabela 4. Índice de correlação de Spearman (rho) entre variáveis motoras e cognitivas. (n=90). Campo Grande/ MS, 2019.

\begin{tabular}{|c|c|c|}
\hline \multirow{2}{*}{ Variáveis motoras } & \multicolumn{2}{|c|}{ Variáveis cognitivas } \\
\hline & MEEM & BAF \\
\hline FES-I & $-0,247^{*}$ & $-0,153$ \\
\hline \multicolumn{3}{|l|}{ TUG simples } \\
\hline Tempo & $-0,031$ & $-0,468 *$ \\
\hline Número de passos & $-0,044$ & $-0,394 *$ \\
\hline \multicolumn{3}{|c|}{ TUG com dupla-tarefa motora } \\
\hline Tempo & $-0,012$ & $-0,475^{*}$ \\
\hline Número de passos & 0,036 & $-0,460 *$ \\
\hline \multicolumn{3}{|c|}{ TUG com dupla-tarefa cognitiva } \\
\hline Tempo & $-0,019$ & $-0,376^{*}$ \\
\hline Número de passos & 0,029 & $-0,361 *$ \\
\hline \multicolumn{3}{|c|}{ Base $30 \mathrm{~cm}$ olhos abertos } \\
\hline Posição AP & 0,027 & 0,212 \\
\hline Posição ML & 0,108 & $-0,240 *$ \\
\hline Área & $-0,081$ & $-0,273^{*}$ \\
\hline Velocidade AP & $-0,166$ & $-0,212$ \\
\hline Velocidade ML & $-0,093$ & $-0,205$ \\
\hline \multicolumn{3}{|c|}{ Base $30 \mathrm{~cm}$ olhos fechados } \\
\hline Posição AP & 0,193 & 0,097 \\
\hline Posição ML & 0,085 & 0,130 \\
\hline Área & 0,117 & $-0,070$ \\
\hline Velocidade AP & 0,096 & $-0,100$ \\
\hline Velocidade ML & 0,027 & $-0,170$ \\
\hline
\end{tabular}


Continuação da Tabela 4

\begin{tabular}{lll}
\hline \multirow{2}{*}{ Variáveis motoras } & Variáveis cognitivas & \\
& MEEM & BAF \\
\hline Base 10 cm olhos abertos & & $-0,231^{*}$ \\
Posição AP & 0,108 & 0,035 \\
Posição ML & 0,004 & $-0,131$ \\
Área & $-0,189$ & $-0,154$ \\
Velocidade AP & $-0,161$ & $-0,143$ \\
Velocidade ML & $-0,213$ & \\
Base 10 cm olhos fechados & & 0,050 \\
Posição AP & $-0,042$ & $-0,257 *$ \\
Posição ML & $-0,074$ & 0,036 \\
Área & $-0,102$ & 0,001 \\
Velocidade AP & $-0,083$ & 0,043 \\
Velocidade ML & $-0,160$ & \\
\hline
\end{tabular}

MEEM: Mini-Exame do Estado Mental. BAF: Bateria de Avaliação Frontal. FES-I: Escala Internacional de Quedas. TUG: Timed Up and Go. AP: Anteroposterior. ML: Médio-lateral. Correlações significativas estão destacadas com asteriscos.

\section{DISCUSSÃO}

Este estudo investigou o impacto da complexidade da tarefa sobre a mobilidade e o equilíbrio de idosos. Os resultados mostraram que, em idosos saudáveis e com baixo risco de quedas, a complexidade da tarefa impactou a mobilidade dos sujeitos, exigindo um maior número de passos e tempo para realizar a atividade. De forma semelhante, base de apoio restrita e informações visuais reduzidas causaram maior insegurança dos participantes para permanecer em posição ortostática. A compreensão desses resultados é importante para o desenvolvimento de terapias capazes de reduzir os riscos à saúde dos idosos.

A meta inicial deste estudo foi incluir um número semelhante de homens e mulheres, a fim de investigar o impacto da complexidade da tarefa nos dois grupos. A realidade observada, contudo, refletiu um número consideravelmente maior de mulheres do que homens. Fatores que podem justificar esse cenário estão relacionados à maior expectativa de vida das mulheres e a tendência de as mulheres serem mais proativas em pesquisas e projetos do que os homens ${ }^{23}$.

Em relação as funções cognitivas, os grupos foram semelhantes para cognição geral, mas divergentes para funções executivas. A BAF difere do MEEM por concentrar sua análise nas funções executivas dos sujeitos, associada principalmente ao córtex pré-frontal ${ }^{24}$. O MEEM, diferentemente, é responsável pela análise geral da cognição e é usado para rastrear casos de demência quando associado à avaliação clínica do paciente ${ }^{25}$. A diferença entre os grupos para funções executivas, embora tenha sido estatisticamente significativa, não é relevante do ponto de vista clínico pois se refere a pontuações normais da BAF segundo valores de referências identificados por Beato et al. ${ }^{17}$. Em adição, essa divergência de escores entre homens e mulheres pode ter sido influenciada pelo grau de escolaridade das mulheres (ligeiramente inferior ao dos homens) e pela rotina das mulheres (vinculada neste estudo às atividades do lar). Como a escala BAF sofre influência de ambos os fatores ${ }^{26,27}$, é provável que esses aspectos tenham impactado o escore final das mulheres sem evidenciar traços de declínio cognitivo.

A maioria dos participantes relatou não ter sofrido quedas nos últimos doze meses. Na escala FES-I, os sujeitos apresentaram escore correspondente a risco esporádico de queda ${ }^{28}$. Mesmo com esses parâmetros, os profissionais de saúde não devem negligenciar idosos com baixo risco de quedas pois os participantes deste estudo, ainda que com quedas infrequentes, sofreram impacto da complexidade da tarefa sobre a sua mobilidade e equilíbrio. 
Os resultados confirmaram a hipótese inicial dos pesquisadores quando demonstrou o impacto da complexidade da tarefa sobre o tempo e o número de passos no teste TUG. Esse resultado já tinha sido observado em estudos prévios ${ }^{29,30}$ e foi confirmado na presente pesquisa. Um fato interessante é que tanto os homens quanto as mulheres apresentaram mais dificuldades em realizar a atividade com dupla-tarefa cognitiva do que a atividade com dupla-tarefa motora. Isso comprova o impacto de situações desafiadoras cognitivas no dia a dia dos idosos - em especial quando o foco da atenção é dividido em mais de uma atividade realizada simultaneamente.

$\mathrm{Na}$ comparação entre sexos, as mulheres precisaram de mais passos para realizar as tarefas de mobilidade do que os homens. Isso não aconteceu com a variável tempo. Diversos fatores podem estar associados a esse achado como tamanho da passada, medo de sofrer quedas e funções cognitivas. Novos estudos devem ser realizados para abordar essa temática e aprofundar a discussão sobre o tópico.

Com relação as medidas estabilométricas, os participantes apresentaram oscilações do equilíbrio quando submetidos a bases de apoio restritas e informações visuais imprecisas. Ambos os fatores impactaram o equilíbrio de idosos saudáveis, corroborando estudos prévios ${ }^{31,32}$. Como baixa visão e bases de apoio instáveis são comuns no envelhecimento, os resultados sugerem o uso de diferentes bases de apoio e dicas visuais durante os protocolos de reabilitação como forma de estimular o idoso a lidar com os desafios motores diários.

Com relação a associação entre variáveis cognitivas e motoras (Tabela 4), o MEEM, que avalia aspectos cognitivos gerais, não foi associado às medidas de mobilidade e equilíbrio. A BAF, diferentemente, mostrou importante associação sobretudo com os testes de mobilidade. Os resultados reforçam a interferência das funções executivas pré-frontais nas atividades motoras diárias dos idosos. Os autores atribuem as poucas e fracas associações entre funções executivas e testes estabilométricos ao fato de a atividade na plataforma de força apresentar restrições físicas (bases de apoio restritas e informações visuais imprecisas) mas com baixa demanda cognitiva.

Ainda que as correlações tenham sido significativas entre funções executiva e mobilidade, as análises demonstraram correlações de fraca $\left(0,10<\left|\mathrm{r}_{\mathrm{s}}\right|<0,39\right)$ e moderada $\left(0,40<\left|\mathrm{r}_{\mathrm{s}}\right|<0,69\right)$ intensidades ${ }^{33}$. Isso demonstra que outros fatores além das funções executivas estão associados à mobilidade dos idosos. Novos estudos devem abordar essa temática e identificar outros fatores que se associam com mobilidade na população em questão.

Os resultados deste estudo devem ser considerados frente a algumas limitações. Primeiramente a amostra foi composta predominantemente por “idosos jovens". Dificuldades na inclusão de idosos com idades mais avançadas estão relacionadas a problemas de locomoção do idoso, altos índices de hospitalização, impossibilidade da pessoa em comparecer ao centro de avaliação e maior prevalência de declínio cognitivo ${ }^{34}$. Em segundo lugar, o número de homens foi significativamente menor do que o número de mulheres. Por fim, as correlações entre testes cognitivos e motores, ainda que significativas, foram de fraca e moderada magnitudes - o que indica que podem haver outros fatores não incluídos nesta pesquisa se associando à mobilidade e ao equilíbrio.

\section{CONCLUSÃO}

Idosos saudáveis apresentam dificuldades de mobilidade e equilíbrio quando submetidos a tarefas complexas. A associação das variáveis cognitivas com as motoras reforça o impacto das funções executivas pré-frontais sobre a mobilidade de idosos, e sugerem a necessidade de reabilitação multiprofissional como forma de estímulo para o idoso enfrentar os desafios diários. Novas pesquisas devem ser realizadas com o objetivo de analisar se há outros fatores impactando a mobilidade e o equilíbrio dos idosos além das variáveis analisadas neste estudo.

Editado por: Daniel Gomes da Silva Machado 


\section{REFERÊNCIAS}

1. Tomioka K, Kurumatani N, Hosoi H. Age and gender differences in the association between social participation and instrumental activities of daily living among community-dwelling elderly. BMC Geriatr. 2017;17(1):1-9. Disponível em: https://doi. org/10.1186/s12877-017-0491-7.

2. Pertl MT, Benke T, Zamarian L, Delazer M. Effects of healthy aging and mild cognitive impairment on a real-life decision-making task. J Alzheimers Dis. 2017;58(4):1077-87. Disponível em: https://doi. org/10.3233/JAD-170119 .

3. Mather M. The emotion paradox in the aging brain. Ann N Y Acad Sci. 2012;1251(1):33-49. Disponível em: https://doi.org/10.1111/j.1749-6632.2012.06471.x .

4. Lee DR, Santo EC, Lo JC, Ritterman Weintraub ML, Patton M, Gordon NP. Understanding functional and social risk characteristics of frail older adults: a crosssectional survey study. BMC Fam Pract. 2018;19(1):19. Disponível em: https://doi.org/10.1186/s12875018-0851-1 .

5. Blazer DG. Cognitive Aging: What we fear and what we know. Perspect Biol Med. 2017;60(4):569 82. Disponível em: https://doi.org/10.1353/ pbm.2017.0043.

6. Connolly D, Garvey J, McKee G. Factors associated with ADL/IADL disability in community dwelling older adults in the Irish longitudinal study on ageing (TILDA). Disabil Rehabil. 2017;39(8):809-16. Disponível em: https://doi.org/10.3109/09638288.20 16.1161848 .

7. Saari T, Hallikainen I, Hänninen T, Räty H, Koivisto A. Relationships between cognition and activities of daily living in Alzheimer's disease during a 5-year follow-up: ALSOVA study. J Alzheimers Dis. 2018;64(1):269-79. Disponível em: https://doi. org/10.3233/JAD-171059 .

8. Delgado C, Vergara RC, Martínez M, Musa G, Henríquez F, Slachevsky A. Neuropsychiatric symptoms in Alzheimer's disease are the main determinants of functional impairment in advanced everyday activities. J Alzheimers Dis. 2019;67(1):381-92. Disponível em: https://doi. org/10.3233/JAD-180771.

9. Thönes S, Falkenstein M, Gajewski PD. Multitasking in aging: ERP correlates of dual-task costs in young versus low, intermediate, and high performing older adults. Neuropsychologia. 2018;119:42433. Disponível em: https://doi.org/10.1016/j. neuropsychologia.2018.09.003 .
10. Laurence BD, Michel L. The fall in older adults: physical and cognitive problems. Curr Aging Sci. 2017;10(3):185-200. Disponível em: https://doi.org/10 .2174/1874609809666160630124552.

11. Marshal F, Susan EF, Paul RM. "Mini-mental state": a practical method for grading the cognitive state of patients for the clinician. J Psychiatr Res. 1975;12(3):189-98. Disponível em: https://doi. org/10.1016/0022-3956(75)90026-6 .

12. Brucki SMD, Nitrini R, Caramelli P, Bertolucci PHF, Okamoto IH. Suggestions for utilization of the mini-mental state examination in Brazil. Arq Neuropsiquiatr. 2003;61(3B):777-81. Disponível em: http://dx.doi.org/10.1590/S0004282X2003000500014.

13. Sanders LMJ, Hortobágyi T, van Gemert SB, van der Zee EA, van Heuvelen MJG. Dose-response relationship between exercise and cognitive function in older adults with and without cognitive impairment: a systematic review and meta-analysis. PLoS ONE. 2019;14(1):e0210036. Disponível em: https://doi.org/10.1371/journal.pone.0210036 .

14. von Elm E, Altman DG, Egger M, Pocock SJ, Gotzsche PC, Vandenbroucke JP, et al.. The Strengthening the Reporting of Observational Studies in Epidemiology (STROBE) statement: guidelines for reporting observational studies. Lancet. 2007;370(9596):1453-7. Disponível em: https://doi. org/10.1136/bmj.39335.541782.AD .

15. Nishadi WV, Pereira AAI, Dhananjaya KDE, Uththamawadu D. An algorithm to construct symmetric latin aquares of order $\mathrm{qn}$ for $\mathrm{q} \geq 2$ and $\mathrm{n} \geq 1$. Am J Eng Res. 2017;6(2):42-50.

16. Dubois B, Slachevsky A, Litvan I, Pillon B. The FAB: a Frontal Assessment Battery at bedside. Neurology. 2000;55(11):1621-6. Disponível em: https://doi. org/10.1212/WNL.55.11.1621 .

17. Beato R, Carvalho VA, Guimarães HC, Tumas V, Souza CP, Oliveira GN, et al. Frontal assessment battery in a Brazilian sample of healthy controls: normative data. Arq Neuropsiquiatr. 2012;70(4):27880. Disponível em: https://doi.org/10.1590/S0004282X2012005000009

18. Podsiadlo D, Richardson S. The timed "Up \& Go": a test of basic functional mobility for frail elderly persons. J Am Geriatr Soc. 1991;39(2):142-8. Disponível em: https://doi. org/10.1111/j.1532-5415.1991.tb01616.x. 
19. Nightingale CJ, Mitchell SN, Butterfield SA. Validation of the Timed Up and Go test for assessing balance variables in adults aged 65 and older. J Aging Phys Act. 2019;27(2):230-3. Disponível em: https:// doi.org/10.1123/japa.2018-0049 .

20. Yardley L, Beyer N, Hauer K, Kempen G, PiotZiegler C, Todd C. Development and initial validation of the falls efficacy scale-international (FES-I). Age Ageing. 2005;34(6):614-9. Disponível em: https://doi. org/10.1093/ageing/afi196.

21. Briggs RC, Gossman MR, Birch R, Drews JE, Shaddeau SA. Balance performance among noninstitutionalized elderly women. Phys Ther. 1989;69(9):748-56. Disponível em: https://doi. org/10.1093/ptj/69.9.748.

22. Osborne JW, Overbay A. The power of outliers (and why researchers should always check for them). Pract Assess Res Eval. 2004;9(6):1-8. Disponível em: https://pareonline.net/getvn.asp?v=9\&n=6.

23. GBD 2016 Mortality Collaborators. Global, regional, and national under-5 mortality, adult mortality, agespecific mortality, and life expectancy, 1970-2016: a systematic analysis for the Global Burden of Disease Study 2016. Lancet. 2017;390(10100):1084-150. Disponível em: https://doi.org/10.1016/S01406736(17)31833-0

24. D’Onofrio G, Panza F, Sancarlo D, Addante F, Solfrizzi V, Cantarini C, et al. Executive dysfunction detected with the Frontal Assessment Battery in Alzheimer's disease versus vascular dementia. J Alzheimers Dis. 2018;62(2):699-711. Disponível em: https://doi.org/10.3233/JAD-170365 .

25. Creavin ST, Wisniewski S, Noel-Storr AH, Trevelyan CM, Hampton T, Rayment D, et al. Mini-Mental State Examination (MMSE) for the detection of dementia in clinically unevaluated people aged 65 and over in community and primary care populations. Cochrane Database Syst Rev. 2016;(1):1-10. Disponível em: https://doi.org/10.1002/14651858.

26. Beato RG, Nitrini R, Formigoni AP, Caramelli P. Brazilian version of the Frontal Assessment Batther (FAB): preliminar data on administration to healthy elderly. Dement Neuropsychol. 2007;1:59-65. Disponível em: http://dx.doi.org/10.1590/S198057642008DN10100010.
27. Hisano S. Relationship between frontal assessment battery scores and activities of daily living/ instrumental activities of daily living ability in older adults. J Phys Ther Sci. 2018;30(10):1237-40. Disponível em: https://doi.org/10.1589/jpts.30.1237.

28. Camargos FFO, Dias RC, Dias JMD, Freire MTF. Cross-cultural adaptation and evaluation of the psychometric properties of the Falls Efficacy Scale - International Among Elderly Brazilians (FESI-BRAZIL). Braz J Phys Ther. 2010;14(3):237-43. Disponível em: http://dx.doi.org/10.1590/S141335552010000300010 .

29. Tomas-Carus P, Rosado H, Pereira C, Marmeleira J, Veiga G, Collado-Mateo D. Differences between two types of dual tasks according to the educational level in older adults. Arch Gerontol Geriatr. 2020;91:e104216. Disponível em: http://dx.doi. org/10.1016/j.archger.2020.104216 .

30. Asai T, Oshima K, Fukumoto Y, Yonezawa Y, Matsuo A, Misu S. Does dual-tasking provide additional value in timed "up and go" test for predicting the occurrence of falls?: a longitudinal observation study by age group (young-older or old-older adults). Aging Clin Exp Res. 2020. Disponível em: http://dx.doi. org/10.1007/s40520-020-01510-6.

31. Lord SR, Clark RD, Webster IW. Visual acuity and contrast sensitivity in relation to falls in an elderly population. Age Ageing. 1991;20(3):175-81. Disponível em: http://dx.doi.org/10.1093/ageing/20.3.175.

32. Polastri PF, Barbieri FA, Brito MB, Bonfim JVA, Gotardi GC, Kuga GK, et al. Semi tandem base of support degrades both saccadic gaze control and postural stability particularly in older adults. Neurosci Lett. 2019;705:227-234. Disponível em: http://dx.doi. org/10.1016/j.neulet.2019.03.004

33. Schober P, Boer C, Schwarte LA. Correlation coefficients: appropriate use and interpretation. IARS. 2018;126(5):1763-8. Disponível em: https://doi. org/10.1213/ANE.0000000000002864 .

34. Nguyen MT, Mattek N, Woltjer R, Howieson $\mathrm{D}$, Silbert L, Hofer S. Pathologies underlying longitudinal cognitive decline in the oldest old. Alzheimer Dis Assoc Disord. 2018;32(4):2659. Disponível em: https://doi.org/10.1097/ WAD.0000000000000265 . 